\title{
A Fuzzy Impulse Noise Filter Based on Boundary Discriminative Noise Detection
}

\author{
Om Prakash Verma* and Shweta Singh*
}

\begin{abstract}
The paper presents a fuzzy based impulse noise filter for both gray scale and color images. The proposed approach is based on the technique of boundary discriminative noise detection. The algorithm is a multi-step process comprising detection, filtering and color correction stages. The detection procedure classifies the pixels as corrupted and uncorrupted by computing decision boundaries, which are fuzzified to improve the outputs obtained. In the case of color images, a correction term is added by examining the interactions between the color components for further improvement. Quantitative and qualitative analysis, performed on standard gray scale and color image, shows improved performance of the proposed technique over existing state-of-the-art algorithms in terms of Peak Signal to Noise Ratio (PSNR) and color difference metrics. The analysis proves the applicability of the proposed algorithm to random valued impulse noise
\end{abstract}

Keywords-Impulse Noise, Decision Boundaries, Color Components, Fuzzy Filter, Membership Function

\section{INTRODUCTION}

Digital images can be contaminated by different types of noises. Impulse noise is one of such noises, which affect images at the time of acquisition due to noisy sensors or at the time of transmission due to channel errors, or in storage media due to faulty hardware. Various detection and filtering algorithms have been proposed. Among them, median filters [1-3] proved to be most prominent in their ability to suppress noise. Presence of three domains of filtering, i.e. spatial, frequency and fuzzy, gives ample opportunity for researchers in image processing to exploit the field of impulse noise filtering. Several linear and non-linear filters have been proposed in the literature. Linear filters are not able to effectively eliminate impulse noise as they can blur the edges. The intuitive solution to overcome the problem of linear filters is to implement an impulse-noise detection mechanism prior to filtering; hence, only those pixels identified as corrupted would undergo the filtering process, while those identified as uncorrupted would remain unchanged. With the incorporation of such a noise detection mechanism into the median filtering framework, the switching median filters [4-6] have shown significant performance improvement. Many non-linear filters based on classical and fuzzy techniques have emerged in recent years. The noise adaptive soft switching median (NASM) filter [7] proposed by Eng et al. consists of a three-level hierarchical noise detection process.

\footnotetext{
Manuscript received September 28, 2012; accepted November 3, 2012.

Corresponding Author: Shweta Singh

* Delhi Technological University, Delhi, India (opverma.dce@gmail.com, singh.shweta1210@gmail.com)
} 
The NASM achieves a fairly robust performance in removing impulse noise, while preserving signal details across a wide range of noise densities, ranging from $10 \%$ to $50 \%$.

The quality of NASM recovered images degrades as noise density increases above $50 \%$. The Boundary Discriminative Noise Detection (BDND) [8], a switching median filter, is proposed for the detection of impulse noise based on a large difference between the noisy pixel and the noise free pixel. This paper claims to achieve better results than NASM by passing the pixel from two sizes of window $(21 \times 21$ and $3 \times 3)$ to confirm whether it is noisy or not. It gives acceptable results up to $80 \%$ of noise density. Srinivasan et al. [9] also presents a new decision based algorithm for restoration of images that are highly corrupted by impulse noise. It removes only the corrupted pixel by the median value or by its neighboring pixel value. Very recently, Tripathi et al. [10] presented a switching median filter which is an advanced boundary discriminative noise detection algorithm. It is also a two stage detection algorithm. Smail Akkoul et al. presented an adaptive switching median filter (ASWM) [11] which requires no prior threshold as required by a classical switching median filter. Threshold is computed locally from image pixels intensity values in a sliding window. Duan and Zhang presented a two iteration algorithm [12] for impulse noise detection for switching median filter.

In the field of fuzzy domain, the fuzzy median filter $[13,14]$ is a modification to the classical median filter. The Fuzzy Inference Rules by Else action (FIRE) filters [15-17] are a family of non-linear operators that adopt fuzzy rules to remove impulse noise from images. Androutsos et al. [18] designed a new class of filters called fuzzy vector rank filters, based on a combination of different distance measures. Stefan et al. [19] presented a fuzzy two-step color filter for the reduction of impulse noise. This filter utilizes the fuzzy gradient values and fuzzy reasoning for the detection of noisy pixels. Verma et al. [20] presents two stage fuzzy filter for reduction of both impulse and Gaussian noise in color images. It also considers the interactions between the color components. Toh at al. presents a cluster based adaptive fuzzy switching median filter [21] for universal impulse noise reduction i.e. random valued or fixed valued impulse noise. Madhu et al. proposed a new efficient fuzzy-based decision algorithm (FBDA) [22] for the restoration of images that are corrupted with high density of impulse noises. Another two phase process of fuzzy logic based impulse noise filtering technique [23] is presented by Aborisade.

Most of the above reported schemes work well under salt and pepper noise but fail under random valued impulse noise, which is more realistic when it comes to real world applications. These schemes generally use a threshold value for identification of pixel as corrupted or uncorrupted. The partitions obtained are thus strict and boundaries are rigid. The fuzzy based boundary discrimination detection algorithm presented here considers smooth boundaries which are computed for each candidate pixel. In the present paper, a two step impulse noise filter is proposed. This approach exploits the advantages of switching median filter and a fuzzy rule based system. The filter works in the stages of detection, filtering and color correction. For gray scale images only the first two are applicable whereas for the color images, the correction term is added after filtering to remove the residue impulse noise remaining in the color components. Simulation results are carried out for random impulse noise, and comparative analysis shows that fuzzifying the decision boundaries gives better performance than the existing techniques in terms of various image metrics.

The paper is divided into four sections. Section 2 discusses the noise models considered for result evaluation. Section 3 presents our Fuzzy Impulse Filter. Section 4 gives the results and comparisons of our approach with existing algorithms. Finally, section 5 concludes the paper. 


\section{NOISE MODELS}

Four impulse noise models are presented in [8]. Out of these four models only two noise models, namely model 3 and 4 , are selected in present case. $I(i, j)$ represents the intensity value at location $i, j$. For the pixel, $y(i, j)$, of noisy image at location $i, j$, the noise models (renamed) are represented as follows:

1) Noise Model 1: An impulse noise in this model is represented with two fixed ranges of length $m$ at both the ends of the gray scale. For example, if $m$ is 20 then the impulse noise used to corrupt an image will have the intensity value in $[0,19]$ and $[236,255]$ with equal probability. The probability density function for $y(i, j)$ in this noise model given as [8]

$$
f(y)= \begin{cases}\frac{p}{2 m}, & 0 \leq y<m \\ 1-p, & y=I(i, j) \\ \frac{p}{2 m}, & 255-m<y \leq 255\end{cases}
$$

where $p$ is the noise density.

2) Noise Model 2: It is an extension of above model with unequal densities of the low intensity and high intensity impulse noises and is given as

$$
f(y)= \begin{cases}\frac{p_{1}}{2 m}, & 0 \leq y<m \\ 1-p, & y=I(i, j) \\ \frac{p_{2}}{2 m}, & 255-m<y \leq 255\end{cases}
$$

where $p=p_{1}+p_{2}$ is the noise density.

\section{FUZZY IMPULSE FILTER}

This section discusses a fuzzy based approach to boundary discriminative noise detection. The proposed algorithm presents an impulse filter which is applicable to both gray scale and color images. The algorithm is a multi step process of detection, filtering and noise correction. Noise correction is specifically designed for color images to remove the residue noise presents in color components by exploiting the interactions between them.

The basic strategy of BDND [8] is to examine each pixel in its neighborhood from coarse to fine. The pixel under consideration is examined in two stages of different window sizes to be marked as corrupted or uncorrupted. The most critical part of this is the determination of the decision boundaries that classifies the pixels as 


$$
\operatorname{Class}(y(i, j))= \begin{cases}\text { Low intensity noise, } & y(i, j) \leq b_{1} \\ \text { Uncorrupted, } & b_{1}<y(i, j) \leq b_{2} \\ \text { High intensity noise, } & y(i, j)>b_{2}\end{cases}
$$

where $y(i, j)$ is the intensity of the pixel being considered, and $b_{1}$ and $b_{2}$ are two decision boundaries. The major weakness of BDND resides in selecting the strict boundaries, which is a reason for misclassification. The issue is resolved by taking the smooth boundaries for the classes (Eq. 3 ) in our improved algorithm for detection. Overlapped regions are treated by creating fuzzy rules to obtain a correct degree of noisyness for the candidate pixel. The detection procedure starts by subjecting a candidate pixel to $21 \times 21$ window centered on it. Decision boundaries are formed similar to [8]. Fuzzification is incorporated in the boundary discriminative noise detection with the help of three membership functions. Functions are described in detail later in this section. Different fuzzy rules are designed to consider all the possibilities of the existence of a pixel in any membership function. Each rule is given a weight, according to which a degree of noisyness is calculated which will decide the pixel is corrupted or uncorrupted. The first part of detection process ends with generating a decision map, with " 0 " representing the corrupted and "1" represents the uncorrupted pixel. The second part of the detection process starts by confirming the pixel's class by imposing it to a $5 \times 5$ window. The decision map formed after this step is given to the filtering stage to filter out the corrupted pixels by replacing them with median of uncorrupted ones only. The output thus obtained is a filtered image and can be considered an output image of fuzzy based filter in case of gray scale images. As mentioned, for color images another step is applied in order to remove leftover impulse noise in color components by checking the difference between the color components and devising a membership function to calculate a correction term to be added to a pixel. The complete algorithm is presented in the following subsections.

\subsection{Noise Detection for gray scale images}

The main aim of the detection step is to classify the pixels as corrupted (high or low intensity) or uncorrupted. Therefore, it is carried out by first finding the decision boundaries. These boundaries are themselves calculated in two iterations i.e. first the pixel are checked on global statistics (21x21 window) and then with local statistics ( $5 \times 5$ window) just to confirm the classification. The boundaries selected are fuzzified in order to avoid the rigidness of the strict thresholds. The output of the detection step is a decision map.

Steps for detection are as follows:

1) Impose $21 \times 21$ window centered on $y(i, j)$

2) Sort the pixels of the window to an ordered vector $v_{o}$ and find the median med.

3) Compute the differences between each pair of adjacent pixels in vector $v_{o}$. The new vector is denoted as $v_{D}$.

4) Find the pixels which correspond to maximum differences in $v_{D}$ corresponding to intervals $\left[0\right.$, med] and (med, 255] in $v_{o}$. And set these two pixels' intensities as the decision boundaries $b_{1}$ and $b_{2}$ respectively.

5) With $b_{1}, b_{2}$ and med values, three membership functions are formed. This step is known as fuzzification of decision boundaries. 


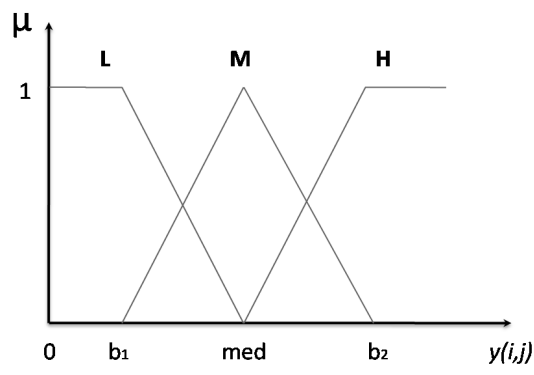

Fig. 1. The membership functions

Three membership functions $\mu_{L}, \mu_{M}$ and $\mu_{H}$ are devised corresponding to a pixel as shown in the Fig. 1. The membership function, $\mu_{L}$ represents a fuzzy set Low (L), indicating the pixels belonging to low intensity corrupted class. Similarly $\mu_{H}$ represents fuzzy set High (H) for high intensity corrupted class. And $\mu_{M}$ is for fuzzy set Medium (M) for pixels which are uncorrupted. The closer the value of a pixel to the boundaries $b_{1}$ and $b_{2}$, the higher is the possibility of a pixel to lie in one of the corrupted classes (low or high).

$$
\begin{aligned}
& \mu_{H}= \begin{cases}1, & y(i, j) \leq b_{1} \\
\frac{m e d}{m e d-b_{1}}-\frac{y(i, j)}{m e d-b_{1}}, & b_{1}<y(i, j) \leq \text { med } \\
0, & \text { otherwise }\end{cases} \\
& \mu_{M}= \begin{cases}1, & b_{1}<y(i, j) \leq b_{2} \\
0, & \text { otherwise }\end{cases} \\
& \mu_{H}= \begin{cases}1, & y(i, j) \leq b_{2} \\
\frac{y(i, j)}{b_{2}-\text { med }}-\frac{\text { med }}{b_{2}-\text { med }}, & \text { med }<y(i, j) \leq b_{2} \\
0, & \text { otherwise }\end{cases}
\end{aligned}
$$

The degree of noise present in the pixel is ascertained by forming fuzzy inference rules. These are used to validate the existence of a pixel in the particular class of output. For a pixel P, the examples of rules are as follows:

Rule 1: If $P$ lies in $L$ and not in $M$ and not in $H$, then $P$ is corrupted (low intensity).

Rule 2: If $P$ lies in $L$ and $P$ lies in $M$ and not in $H$, then $P$ is corrupted (low intensity) or uncorrupted.

Rule 3: If the pixel lies in $M$ and $P$ lies in $H$ and not in $L$, then $P$ is uncorrupted or corrupted (high intensity).

Each rule has a participation weight, $N_{x}$ assigned to it. The expression of $N_{x}$ is obtained by modifying the weight presented in [26]. 


$$
N_{x}=\frac{l+m+h}{n(n-1)}
$$

where $l, m$ and $h$ represents the index numbers of $\mu_{L}, \mu_{M}$ and $\mu_{H}$ in each rule and $n$ is 3 in present case. The pixels are classified into corrupted and uncorrupted classes by obtaining the degree of noisyness given as [27]

$$
N=\frac{\sum_{z} N_{x} \mu_{p r e m}^{x}}{\sum_{z} \mu_{p r e m}^{x}}
$$

where $z$ is the number of rules (here 27), $N_{x}$ is the weight of each premise and $\mu_{p r e m}^{x}$ is a certainty of premise for the $x^{\text {th }}$ rule. The certainty of premise is obtained by applying the Mamdani fuzzy rule on membership functions

$$
\mu_{\text {prem }}^{x}=\mu_{L} \wedge \mu_{M} \wedge \mu_{H}
$$

The pixel is classified into the particular output class by following the equation

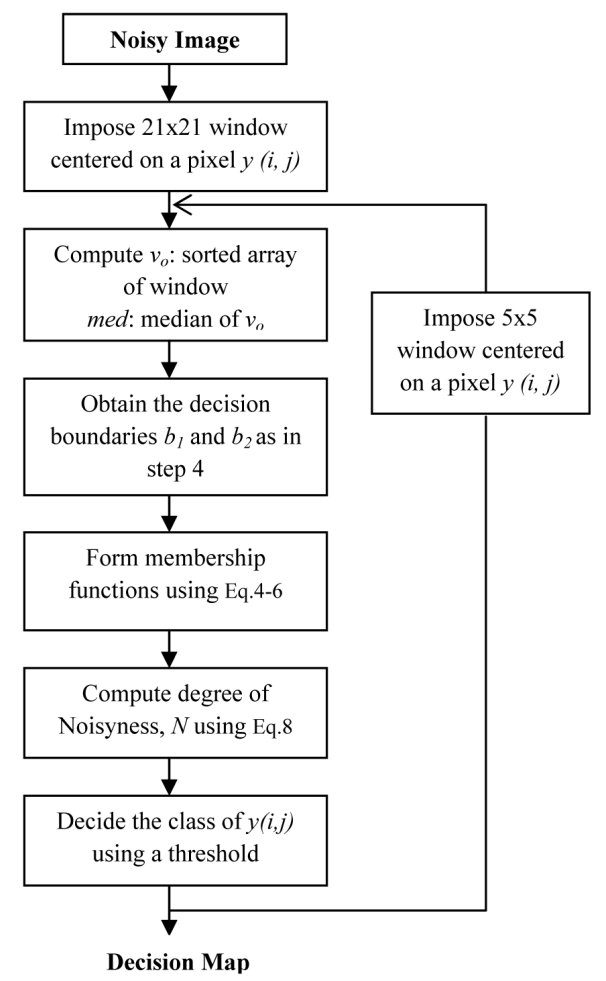

Fig. 2. Flow chart for detection stage 


$$
\text { class }= \begin{cases}\text { uncorrupted, } & N \leq \text { Threshold } \\ \text { corrupted, } & N>\text { Threshold }\end{cases}
$$

The value of threshold is experimentally computed.

6) The validation of noisy candidates is confirmed by imposing a $5 \times 5$ window and repeating the steps $2-5$.

Fig. 2 shows the complete flow chart to depict the above steps.

\subsection{Filtering}

The decision map obtained in the previous section is given as an input to this stage. Only the uncorrupted pixels are selected for filtering i.e. the pixels represented as " 0 " in the decision map. The important change in the filtering technique is the approach of incremental window size [7, 8]. The window size is increased from $3 \times 3$ to $7 \times 7$ depending on the criterion that number of uncorrupted pixels should be more than or equal to half of the total number of pixels in that particular window i.e.

$$
\text { No. of uncorrupted pixels in a window } \geq \frac{1}{2}(W \times W)
$$

where $\mathrm{W}$ is window size. If the above condition holds true then the median value is assigned otherwise $\mathrm{W}$ is incremented by 1 .

The window size is limited to $7 \times 7$ because for larger windows severe blurring takes place in high density noise. The median of the particular window is assigned to the pixel. The filtered image $\boldsymbol{F}$ obtained is considered as an output of the gray scale version whereas for the color version, $\boldsymbol{F}$ is subjected to noise correction.

\subsection{Noise Correction for Color Images}

The noise detection in color images is performed on the same lines as that of gray scale images. The membership functions are formed for each color component. The filtered image $\boldsymbol{F}$ obtained from the filtering step is subjected to this correction step. This filter invokes the interaction between the color components [20] to remove further left-over impulse noise present in color components. The most widely used RGB color space will be used in our work. Here the pixel is represented as $\boldsymbol{F}(\boldsymbol{i}, \boldsymbol{j}, \boldsymbol{z})$ where $\mathrm{z}$ ranges from 1 to 3 representing 1 for the red, 2 for the

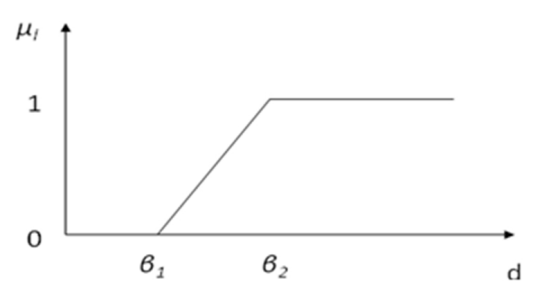

Fig. 3. The membership function "large" 
green, and 3 for the blue component. The correction term to be added to the components is decided by examining the differences in each color pair and forming a membership function. The steps [20] are as described below.

1) Differences between the color pairs is calculated to check for any residual impulse noise in individual color components:

$$
\begin{aligned}
& d_{r g}(i, j)=|F(i, j, 1)-F(i, j, 2)| \\
& d_{r b}(i, j)=|F(i, j, 1)-F(i, j, 3)| \\
& d_{g b}(i, j)=|F(i, j, 2)-F(i, j, 3)|
\end{aligned}
$$

where $d_{r g}(i, j), d_{r b}(i, j), d_{g b}(i, j)$ represent differences between red-green, red-blue and green-blue components for the same pixel of the filtered image F.

2) A fuzzy rule system is framed to compute the degree of noise present in the color component of the pixel concerned. For each component the rule of the following form is formed:

Rule: If $d_{r g}(i, j)$ is Large and $d_{g b}(i, j)$ is Large, then the green component is noisy.

Similar fuzzy rules are coined for other color components. The significance of this rule holds only when there is an impulse noise left in the color components. The application of this stage nullifies when there is region of same color as the differences will be large for that region. Let us say there is a green region present in an image; this step will not consider this area as noisy as the median of the region will also be green. Thus it makes the filter more efficient with respect to noises present in the color components. The definition of "Large" is expressed by the membership function $\mu_{l}$ with the parameters $\beta_{1}$ and $\beta_{2}$ as shown in Fig. 3. For every difference computed above (generalized as $\mathrm{d}$ ), $\mu_{l}$ is given as

$$
\mu_{l}= \begin{cases}0, & d<\beta_{1} \\ \frac{d}{\beta_{2}-\beta_{1}}-\frac{\beta_{1}}{\beta_{2}-\beta_{1}}, & \beta_{1}<d \leq \beta_{2} \\ 1, & d \geq \beta_{2}\end{cases}
$$

3) The degree of noise, $n_{d}$ in the color component is obtained by the minimum value of the membership functions corresponding to the differences. The correction term is given by:

$$
\Delta(i, j, z)=n_{d}(i, j, z) \times(\operatorname{med}(i, j, z)-F(i, j, z))
$$

4) The final output of the impulse filter is given by:

$$
O(i, j, z)=F(i, j, z)+\Delta(i, j, z)
$$

Fig. 4 shows the flow chart representing the second stage of the proposed algorithm. 


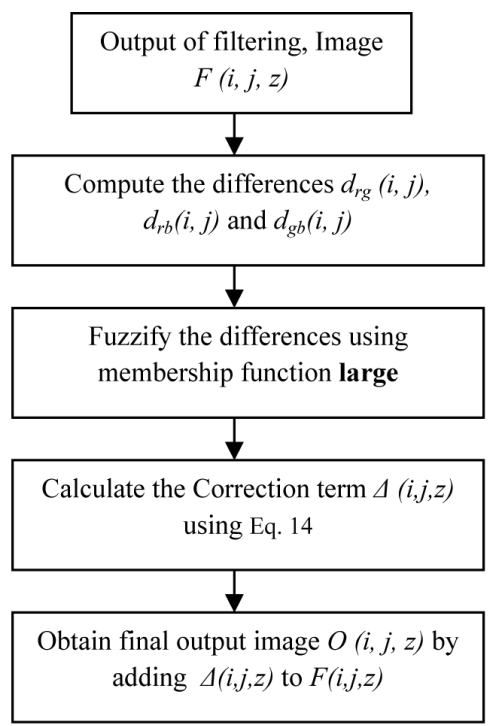

Fig. 4. Flow chart of Noise Correction

\section{Results And Comparative Analysis}

The simulation results are obtained both on gray scale and color images. The proposed approach is compared with four existing algorithms, ASWM [11], BDND [8], SMF [10] and DBAIN [9]. The bases for comparisons are image metrics which are selected according to the type of an image. All the simulations are carried out with Matlab 2010b on Intel Core 2 Duo CPU of $2.53 \mathrm{GHz}$ speed and 1.99 GB RAM machine.

\subsection{Gray Scale Images}

The performance evaluation of the filtering operation is quantified by PSNR. For an image of size $\mathrm{MxN}$

$$
P S N R=10 \log _{10}\left(\frac{255^{2}}{M S E}\right) d B
$$

The filtered image $\boldsymbol{F}$ of each algorithm is compared with that of proposed approach. For experiments, gray scale image of Lena of size $256 \times 256$ is selected. Two noise models are implemented as discussed in section II to compare the results. To study the quantitative performance of algorithms, an input image is corrupted with impulse noise with different densities. Here the results are shown to compare the technique with ASWM (initial alpha=20, iterations=7), BDND (two stages of $21 \times 21$ and $3 \times 3$ with adaptive filtering), SMF (21x21 window with four directional kernels using the thresholds values as $5 / 255$ and $1 / 255)$ and DBAIN ( $3 \times 3$ window). Table 1 (a) and (b) shows the values of PSNR for all the five algorithms for noise density 50\%. Fig 5(a) compares the algorithms graphically. 
Table 1. Quantitative performance (in terms of PSNR values) on Lena (gray scale)

(1) Noise Model 1

\begin{tabular}{c|c|c|c|c|c|c}
\hline \multicolumn{2}{c|}{ Noise=50\% } & \multirow{2}{*}{ ASWM } & \multirow{2}{*}{ BDND } & \multirow{2}{*}{ SMF } & \multirow{2}{*}{ DBAIN } & \multirow{2}{*}{ Proposed } \\
\cline { 1 - 2 } Low Range & High Range & & & & & \\
\cline { 1 - 2 }$[\mathbf{0 , 9}]$ & {$[\mathbf{2 4 6 , 2 5 5}]$} & 21.42 & 24.34 & 25.20 & 9.90 & 25.72 \\
\hline$[\mathbf{0 , 1 9}]$ & {$[\mathbf{2 3 6 , 2 5 5}]$} & 21.19 & 24.31 & 23.99 & 9.53 & 25.31 \\
\hline$[\mathbf{0 , 2 9}]$ & {$[\mathbf{2 2 6 , 2 5 5}]$} & 21.01 & 22.85 & 21.05 & 9.37 & 23.91 \\
\hline$[\mathbf{0 , 3 9}]$ & {$[\mathbf{2 1 6 , 2 5 5}]$} & 21.06 & 18.84 & 18.86 & 9.03 & 22.32 \\
\hline$[\mathbf{0 , 4 9}]$ & {$[\mathbf{2 0 6 , 2 5 5}]$} & 20.22 & 16.02 & 15.26 & 8.86 & 21.85 \\
\hline
\end{tabular}

(2) Noise Model 2

\begin{tabular}{|c|c|c|c|c|c|c|c|c|c|c|c|}
\hline \multicolumn{2}{|c|}{ Noise $=50 \%$} & \multirow{2}{*}{\multicolumn{5}{|c|}{$\mathbf{m}=\mathbf{1 0}$}} & \multirow{2}{*}{\multicolumn{5}{|c|}{$\mathbf{m}=\mathbf{3 0}$}} \\
\hline \multirow{2}{*}{$\begin{array}{c}\text { Low } \\
\text { Density }\end{array}$} & \multirow{2}{*}{$\begin{array}{c}\text { High } \\
\text { Density }\end{array}$} & & & & & & & & & & \\
\hline & & ASWM & BDND & SMF & DBAIN & Proposed & ASWM & BDND & SMF & DBAIN & Proposed \\
\hline 10 & 40 & 20.01 & 25.95 & 26.97 & 10.81 & 28.56 & 20.70 & 24.02 & 22.32 & 11.46 & 25.66 \\
\hline 20 & 30 & 16.53 & 24.17 & 26.05 & 9.55 & 26.73 & 18.84 & 23.53 & 21.92 & 10.29 & 24.17 \\
\hline 30 & 20 & 15.44 & 25.59 & 26.06 & 8.83 & 26.37 & 16.62 & 20.01 & 20.00 & 9.40 & 22.01 \\
\hline 40 & 10 & 18.80 & 25.88 & 27.69 & 9.90 & 28.13 & 20.58 & 22.92 & 22.80 & 10.23 & 23.19 \\
\hline
\end{tabular}

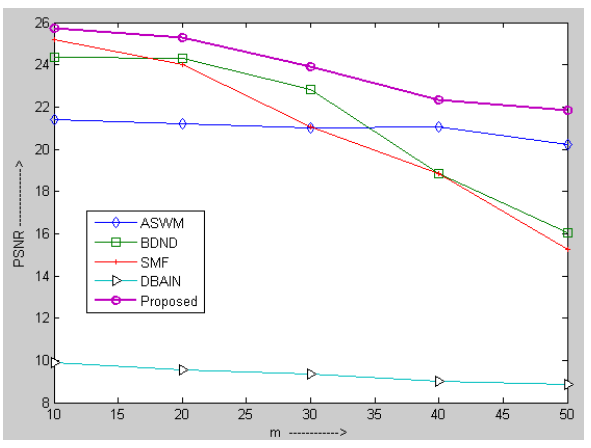

(1) m versus PSNR for gray scale image

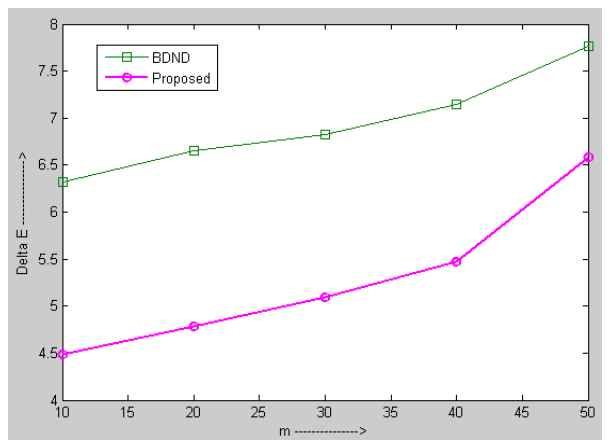

(2) $\mathrm{m}$ versus $\Delta \mathrm{E}$ for color image

Fig. 5. Graphical comparison for noise model 1 on Lena

In the Noise Detection step, the membership functions are formed adaptively according to the value of pixel in hand. That pixel is classified, after fuzzification, into a corrupted or uncorrupted class using a threshold value of Eq. 10. Experimentally the value of threshold for which the technique gives best results is 0.5 .

\subsection{Color Images}

PSNR is just a numerical value more applicable to the monochrome images, thus we need a metric which can appropriately measure the differences between the color images. In response to this need, the perceptually uniform color space CIELAB, standardized by the Commission Internationale de l'Eclairage (CIE) [24], is more accurate for defining quantitative measurements 
Table 2. Quantitative performance (in terms of mean value of $\Delta \mathrm{E}$ ) on Lena (Color)

(1) Noise Model 1

\begin{tabular}{c|c|c|c}
\hline \multicolumn{2}{|c|}{ Noise=40\% } & \multirow{2}{*}{ BDND } & \multirow{2}{*}{ Proposed } \\
\hline Low Range & High Range & 6.32 & 4.49 \\
\hline$[\mathbf{0 , 9}]$ & {$[\mathbf{2 4 6 , 2 5 5}]$} & 6.65 & 4.78 \\
\hline$[\mathbf{0 , 1 9}]$ & {$[\mathbf{2 3 6 , 2 5 5}]$} & 6.82 & 5.09 \\
\hline$[\mathbf{0 , 2 9}]$ & {$[\mathbf{2 2 6 , 2 5 5}]$} & 7.15 & 5.47 \\
\hline$[\mathbf{0 , 3 9}]$ & {$[\mathbf{2 1 6 , 2 5 5}]$} & 7.77 & 6.58 \\
\hline$[\mathbf{0 , 4 9}]$ & {$[\mathbf{2 0 6 , 2 5 5}]$} & &
\end{tabular}

(2) Noise Model 2

\begin{tabular}{c|c|c|c|c|c}
\hline \multicolumn{2}{c|}{ Noise=40\% } & \multicolumn{2}{|c}{ m=10 } & \multicolumn{2}{c}{ m=30 } \\
\cline { 1 - 5 } $\begin{array}{c}\text { Low } \\
\text { Density }\end{array}$ & $\begin{array}{c}\text { High } \\
\text { Density }\end{array}$ & BDND & Proposed & BDND & Proposed \\
\cline { 4 - 6 } & $\mathbf{3 0}$ & 5.84 & 3.73 & 6.58 & 4.86 \\
\hline $\mathbf{1 0}$ & $\mathbf{2 0}$ & 10.10 & 5.09 & 11.80 & 6.18 \\
\hline $\mathbf{3 0}$ & $\mathbf{1 0}$ & 6.15 & 3.758 & 7.20 & 4.40 \\
\hline
\end{tabular}

of perceptual error between the two color vectors. In CIELAB color space the color difference is calculated in terms of $\Delta \mathrm{E}$. It is given by [25]:

$$
\Delta E=\sqrt{\left(L_{2}^{*}-L_{1}^{*}\right)^{2}+\left(a_{2}^{*}-a_{1}^{*}\right)^{2}+\left(b_{2}^{*}-b_{1}^{*}\right)^{2}}
$$

where $\left(L_{1}, a_{1}, b_{1}\right)$ and $\left(L_{2}, a_{2}, b_{2}\right)$ are lab transform of the RGB image. Here we will consider the average value of $\Delta \mathrm{E}$ for complete image.

Table 2 (a) and (b) shows the comparison of BDND with the proposed approach in terms of color difference on color image of Lena of size $256 \times 256$. According to the definition of $\Delta \mathrm{E}$, the value of 1 means there are almost no perceptible differences or variations between two colors. The value ranging from 2-5 represents minute and 6-10 represents noticeable color differences or variations in high quality imaging systems. According to the values obtained for different noise models, our approach shows an acceptable range as compared to BDND. Fig 5(b) shows the comparison graphically. The incorporation of the second stage i.e. to add correction term to color components by exploiting the interactions between them proves to be beneficial. The parameters $\beta_{1}$ and $\beta_{2}$ used are found experimentally to be 0.5 and 0.6 respectively.

\subsection{Visual Performances}

As a final illustration and comparative analysis, Fig 6 and 7 shows the filtered and output images for gray scale and color versions of the input Lena image respectively for specified noise densities. The algorithm performs well for generalized models of impulse noise.

For gray scale, the proposed algorithm is being compared with all the four existing algorithms. As shown ASWM, BDND and SMF give comparable results for noise model 1 with $m=10$ but DBAIN does not perform well as it was only defined for salt and pepper noise. In the color version, the proposed algorithm is compared with BDND only. 


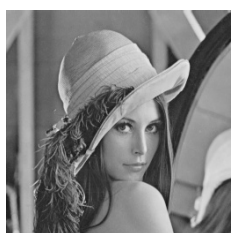

(1) Original

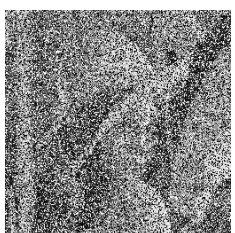

(2) Noisy

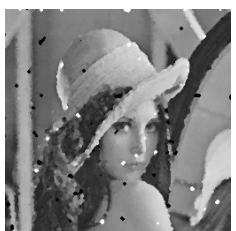

(3) ASWM

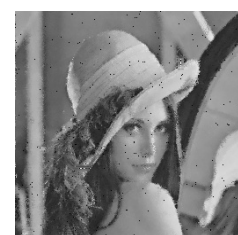

(4) BDND

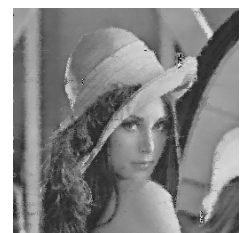

(5) SMF

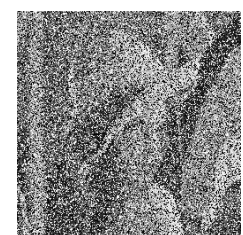

(6) DBAIN

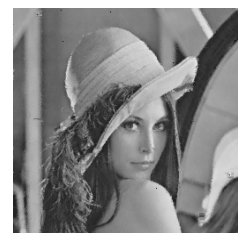

(7) Proposed

Fig. 6. Outputs of different methods for Lena (gray scale) image for noise model 1 ( $50 \%$ impulse noise density, $\mathrm{m}=10$ )

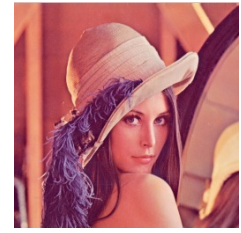

(1) Original

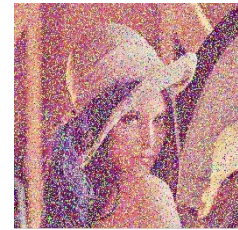

(2) Noisy

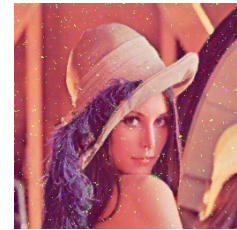

(3) BDND

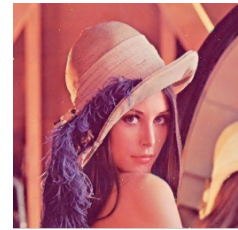

(4) Proposed

Fig. 7. Outputs for Lena (color) image for noise model 2 ( $40 \%$ impulse noise density \{high density=30, low density $=10\}, m=30$ )

\section{CONCLUSION}

We have presented a multi-step fuzzy approach to a boundary discriminative noise detection algorithm for both gray scale and color images. The pixels are classified by forming the fuzzy rules based on the membership functions of decision boundaries obtained using a boundary discriminative approach. The major modification in the technique is incorporated by considering the interactions between the color components. Our approach considers both kinds of interactions i.e. between the same components (e.g. R-R, G-G in the detection step) and between different components (e.g. R-G, G-B and R-B in the color correction step). Thus an additional stage for color images to consider the interaction between the color components removes any residual noise present in the color components after filtering. The quantitative, qualitative and graphical comparison in terms of PSNR and average value of $\triangle \mathrm{E}$ proves that the proposed algorithm performs well as compared to other existing algorithms. From extensive experimentation of the proposed algorithm, it was found that it tends to give a blurred image over $50 \%$ noise density, but the quality of the output images were under considerable limits as represented by $\Delta \mathrm{E}$ value. As shown, using two kinds of noise model, our approach is more suitable for random impulse noise. The proposed work can be extended to include other types of noise like Gaussian or mixed noise. 


\section{REFERENCES}

[1] I. Pitas and A. N. Venetsanopoulos, "Order statistics in digital image processing," Proc. IEEE, Vol.80, No.12, December, 1992, pp.1893-1921.

[2] D. R. K. Brownrigg, "The weighted median filter," Commun. ACM, Vol.27, No.8, August, 1984, pp.807-818.

[3] S.-J. Ko and Y. H. Lee, "Center weighted median filters and their applications to image enhancement," IEEE Trans. Circuits Syst., Vol.38, No.9, September, 1991, pp.984-993.

[4] H. Hwang and R. A. Haddad, “Adaptive median filters: New algorithms and results, " IEEE Transaction Image Processing, Vol.4, No.4, April, 1995, pp.499-502.

[5] Z. Wang and D. Zhang, "Progressive switching median filter for the removal of impulse noise from highly corrupted images, ” IEEE Trans. Circuits Syst. II, Vol.46, No.1, January, 1999, pp.78-80.

[6] S. Zhang and M. A. Karim, “A new impulse detector for switching median filters, ” IEEE Signal Processing Letter, Vol.9, No.4, November, 2002, pp.360-363.

[7] H.-L. Eng and K.-K. Ma, "Noise adaptive soft-switching median filter," IEEE Transaction Image Processing, Vol.10, No.2, February, 2001, pp.242-251.

[8] P.-E. Ng and K.-K. Ma, “A switching median filter with boundary discriminative noise detection for extremely corrupted images,” IEEE Transaction Image Processing., Vol.15, No.6, Junuary, 2006, pp.1506-1516.

[9] K.S. Srinivasan, D.Ebenezer, “A New Fast and Efficient Decision Based Algorithm for removal of High Density Impulse Noises”, IEEE Signal Processing Letters, Volume 14, Issue 3, 2007, pp.189-192.

[10] Tripathi, Ghanekar, Mukhopadhyay, "Switching Median Filter: advanced boundary discriminative noise detection algorithm”, Image Processing, IET, Volume 5 Issue 7, 2011, pp.598-610.

[11] Smail Akkoul, Roger Ledee, Remy Leconge and Rachid Harba, "A New Adaptive Switching Median Filter”, IEEE Signal Processing Letters, 2010, pp 587-590.

[12] Fei Duan and Yu-Jin Zhang, "A Highly Effective Impulse Noise Detection Algorithm for Switching Median Filter”, IEEE Signal Processing Letters, Vol.17, No.7, July, 2010, pp.647-650.

[13] K. Arakawa, "Median Filter based on Fuzzy Rules and its Application to Image Restoration”, Fuzzy Sets and Systems, Vol.77, Issue 1, January, 1996, pp.3-13.

[14] K. Arakawa, "Fuzzy-Rule Based Image Processing with Optimization”, in "Fuzzy Techniques in Image Processing”, E.E. Kerre, M. Nachtegael (Editors), Physica-Verlag, Heidelberg, New York, 2000, pp.222-247.

[15] F. Russo, "FIRE operators for Image Processing", Fuzzy Sets and Systems, Vol.103, 1997, pp.265275.

[16] F. Russo, "Noise Cancellation using Non-linear Fuzzy Filters”, Proc. of IEEE Instrumentation and Measurement Technology Conference, May, 1997, pp.772-777.

[17] F. Russo and G. Ramponi, “A noise smoother using cascaded FIRE filters”, Proc. of 4th Intl. Conf. on Fuzzy Systems, Vol.1, 1995, pp.351-358.

[18] D. Androutsos, K.N. Plataniotis, and A.N. Venetsanopoulos, "Color image processing using vector rank filters”, International Conf. on Digital Signal Processing, Vol.2, 1995, pp.614-619.

[19] S. Schulte, V. De Witte, M. Nachtegael, D. Van der Weken, and E.E. Kerre, "Fuzzy Two-Step Filter for Impulse Noise Reduction From Color Images”, IEEE Trans. on Image Processing, Vol.15, No.11, November, 2006, pp.3567-3578.

[20] Om Prakash Verma, Madasu Handmandlu, Anil Singh Parihar, Vamsi Krishna Madasu, "Fuzzy Filters for Noise Reduction in Color Images”, ICGST-GVIP Journal, Volume 9, Issue 5, ISSN: $1687-$ 398X., September, 2009, pp.29-43.

[21] K.K.V. Toh, N.A.M. Isa, "Cluster-based adaptive fuzzy switching median filter for universal impulse noise reduction”, IEEE Transactions on Consumer Electronics, Volume 56, Issue 4, 2010, pp.25602568.

[22] Madhu S. Nair, G. Raju, “A new fuzzy-based decision algorithm for high-density impulse noise removal”, Signal, Image and Video Processing, 2010 Springer-Verlag. DOI:0.1007/s11760-010-0186-4.

[23] Aborisade, D.O., "A Novel Fuzzy Logic Based Impulse Noise Filtering Technique”, International Journal of Advanced Science and Technology, Vol.32, July, 2011, pp.79-88. 
[24] Werner Backhaus, Reinhold Kliegl, "Color Vision: Perspective from Different Disciplines", John Simon Werner, 1998.

[25] M. Tkalcic and J. F. Tasic, "Color spaces: Perceptual, historical and application background", in Proceeding s of IEEE EUROCON, Vol.1, September, 2003, pp.304-308.

[26] Recep Demirci, "Rule-based automatic segmentation of color images", AEU-International Journal of Electronics and Communications, Volume 60, Issue 6, 2006, pp 435-442.

[27] R. Demirci , Ugur Güvenç, "Fuzzy filter for color images", 1st international fuzzy systems Symposium, Ankara, October, 2009, pp.365-370.

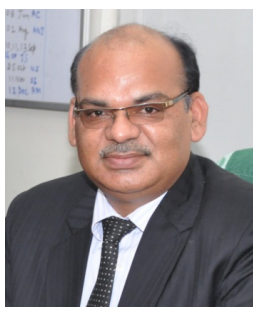

\section{Om Prakash Verma}

Om Prakash Verma received his B.E. degree in Electronics and Communication Engineering from Malaviya National Institute of Technology, Jaipur, India, M. Tech. degree in Communication and Radar Engineering from Indian Institute of Technology (IIT), Delhi, India and Ph.D. degree from Delhi University. From 1992 to 1998 , he worked at Department of Electronics \& Communication Engineering at Malaviya National Institute of Technology, Jaipur, India as an Assistant Professor. He joined Department of Electronics \& Communication Engineering, Delhi Technological University (Formerly Delhi College of Engineering), Delhi, India, as an Associate Professor in 1998. Presently, he is Professor and Head, Department of Information Technology at Delhi Technological University. He has authored a book on Digital Signal Processing in 2003. His research interests include Computer Vision and Image Processing, Application of Soft Computing techniques in Image Processing, Artificial Intelligence, Optimization techniques, and Digital Signal Processing. He has published 35 research papers in International Journal and conference proceedings. He has guided $30 \mathrm{M}$. Tech. students, and presently 5 Ph.D. scholars are working under his supervision. He is Principal investigator of the "Information Security Education Awareness" Project, sponsored by the Department of Information Technology, Ministry of MHRD, Government of India.

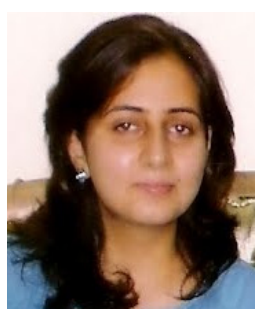

\section{Shweta Singh}

Shweta Singh received her B.Tech. degree in Computer Science and Engineering from Indira Gandhi Institute of Technology, Guru Gobind Singh Indrapastha University, Delhi, India in 2009 and M. Tech. degree in Computer Technology and Applications from Delhi Technological University (Formerly Delhi College of Engineering), Delhi, India in 2012. She joined the Department of Information Technology, Delhi Technological University in 2009 as an Assistant Professor and worked there for 2 years. Her areas of research include Computer Vision, Image Processing and Game theoretic optimization, and watermarking techniques. 